\title{
La historia desconocida tras el logo de la Sociedad Chilena de Pediatría: Il Bambino de L'ospedale degli Innocenti
}

\author{
FRANCISCO MORAGA M. ${ }^{1}$
}

1. Servicio de Pediatría, Complejo Hospitalario San Borja-Arriarán. Director Sociedad Chilena de Pediatría.

\section{ABSTRACT \\ The unknown history behind the Chilean Pediatric Society logo: Il Bambino de L'ospedale degli Innocenti}

There are two kinds of logo standard: a name or a name and drawing and its objective is to capture the essence of the organization that they represent. The logo of the Chilean Society of Pediatrics (SOCHIPE) is a graphic expression of glaced terracottas made by Andrea Della Robbia in 1463, situated at the Hospital of the Innocents in Florence, the first institution devoted to host, care and education of abandoned children in Europe, whose image shows the reception of the child abandoned at the wheel revolving door, allowing anonymously that abandoned children were recepted for their care and social reintegration. The construction of the hospital by Brunellesco also marks the beginning of the Renaissance architecture, a new conception of forms and spaces for institucionalized children and the establishment of the concept of fraternal help, beyond simple charity, to the child in biological, psychological and social distress, being the first active action by the authorities. SOCHIPE, in the First Article of its Statutes, manifested its intention to promote the rights and welfare of children and adolescents through many initiatives. So, our logo captures and proyects this renaissance conception of the child as a subject of law that, far from being changed, is actually more valid, considering all the situations that interact in current times with the commitment to protect children rights.

(Key words: Logo, della Robbia, child rights, Hospital of the Innocents, orphans, child health, history of medicine, childhood protagonism).

Rev Chil Pediatr 2008; 79 (2): 190-198

\section{RESUMEN}

Existen dos clases de logotipos estándar: un nombre, o un nombre y un dibujo; su fundamento es capturar la esencia de la organización a la que representa. El logo de la Sociedad Chilena de Pediatría (Sochipe) es la expresión gráfica de las terracotas confeccionadas por Andrea Della Robbia en 1463, ubicadas en el Hospital de los Inocentes en Florencia, primea institución dedicada a acoger, cuidar, restituir la salud y 
educar a los niños abandonados en Europa, cuya imagen representa la acogida del menor abandonado en la rueda que, junto con la inscripción ubicada en el pórtico del Hospital, permitía que, anónimamente, los niños abandonados fueran depositados y recepcionados para su atención y reinserción social. La construcción del Hospital por parte de Brunellesco marca también el inicio del Renacimiento arquitectónico, una nueva concepción de formas y espacios para los niños institucionalizados y la aparición del concepto de ayuda fraterna, más allá de la simple caridad, para con el niño en situación de desamparo biológico, psicológico y social en lo que constituye la primera acción decidida por autoridades destinada a la protección del Derecho a la Vida de los niños. La Sochipe, en el Primer Artículo de sus Estatutos, destaca su compromiso de promover los derechos y el bienestar del niño y adolescente, a través de múltiples iniciativas; así, nuestro logo recoge y proyecta esta naciente concepción renacentista del niño como sujeto de derecho la que, lejos de haber sufrido cambios, se encuentra más vigente que nunca, considerando el sinnúmero de situaciones que los tiempos actuales interactúan poniendo en peligro el compromiso de proteger los derechos de los niños.

(Palabras clave: logotipo, della Robbia, derechos del niño, Hospital de los Inocentes, huérfanos, salud infantil, historia de la medicina, protagonismo infantil).

Rev Chil Pediatr 2008; 79 (2): 190-198

\section{Introducción}

La función de un logotipo consiste en capturar la esencia de una organización en una imagen, por lo que generalmente incluyen un nombre y un símbolo para hacerlos más distinguibles. Un buen logotipo debe ser simple -con pocas imágenes, colores o fuentes-, único -para que pueda ser reconocido con facilidad-y flexible, para que pueda ser impreso en diversos colores y tamaños en una gran variedad de superficies. El Logo es un elemento que representa constantemente a una organización y es por eso que las grandes empresas no lo cambian casi nunca y si lo hacen, es esporádicamente y con mínimas modificaciones, como por ejemplo los logos de IBM, que desde hace más de 70 años casi no ha tenido modificaciones o el de Coca Cola, casi igual desde $1886^{1,2}$.

El logotipo de la Sociedad Chilena de Pediatría (SOCHIPE), la imagen de un niño envuelto en géneros desde el tronco a los pies, con gesto especial y las palmas en actitud de súplica sobre un fondo tricolor festoneado, ha acompañado a sus socios por más de medio siglo; proviene de uno de los 14 (originalmente 10) bajorrelieves, de terracota vidriada esculpidos por Andrea Della Robbia en 1446, que adornan el Hospital de los Inocentes u "Ospedale degli Innocenti" localizado en la "Piazza della SS. Annunziatia" en Florencia, Italia.
La fecha exacta en que logotipo de la SOCHIPE hace su aparición oficial está en las sombras de los tiempos; sin embargo, debemos ubicarlo entre 1922, fecha de la constitución de la Sociedad Chilena de Pediatría ${ }^{3}$, e inicios de 1926 donde lo encontramos impreso por primera vez en el Anuario 1925 del Hospital de Niños Manuel Arriarán (figura 1), siendo subdirector y Médico Jefe el Dr. Alfredo Comentz, a la fecha miembro del Directorio y ex Vicepresidente de la Sociedad Chilena de Pediatría², en tiempos en que la Academia y lo asistencial eran inseparables.

Coincidentemente, la Academia Americana de Pediatría (AAP), en 1930, propone un logo semejante, que se modifica en 1940, haciéndose oficial sólo desde el año 1955 (figura 2) ${ }^{4,5}$. La Sociedad Brasileña de Pediatría, fundada el 27 de Julio de 1910, también incorpora un logo semejante en el año 1936, sufriendo leves modificaciones en los años 1957 y 1968 (figura 2). En ambas instituciones, el origen declarado del logo es el mismo, según lo relatan Beaven ${ }^{5}$ para la AAP y en su página web, en la sección Historia de la Pediatría Brasileña (http://www. sbp.com.br/), respectivamente. En todos los casos, la imagen de este "Jesús-niño" representa la intención de protección a la infancia en desamparo. Cabe destacar que para esas fechas, nuestro país era parte de la sección latinoamericana de la AAP, o IX Distrito, el que a 
su vez comprendía 4 zonas o presidencias: zona sur, zona brasileña, zona bolivariana y Centroamérica; las reuniones eran periódicas y cabe suponer que los logos fueran conocidos por los otros integrantes de este IX Distrito así como por parte de la Directiva central en Estados Unidos ${ }^{6}$.

En Mayo de 1955 asiste como invitado de honor a nuestro país el entonces Presidente de la AAP Harry Bakwin (1893-1973), notable pediatra y coleccionista de arte, quien recientemente había publicado junto a su esposa Ruth Bakwin, el clásico "Clinical Manegment of Behavior Disorders in Children” (Philadelphia, WB Saunders $1^{\circ}$ Ed. 1953), y quién fue el

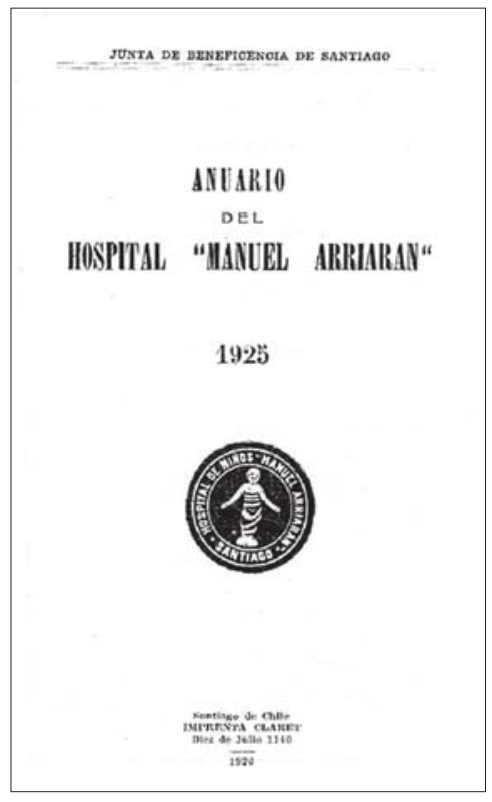

Figura 1. Primera publicación conocida que incorpora los diseños iniciales del Logo. encargado de aprobar oficialmente el actual logo de la AAP el 29 de Septiembre de 1955; fue nominado Miembro Honorario de la Sociedad Chilena de Pediatría y dictó clases magistrales sobre "Hospitalismo" y otros temas relacionados a los Derechos de los Niños ${ }^{7}$. En 1956 fue presidente del IX Distrito el Prof. Dr. Aníbal Ariztía, mientras que era Delegado por la Rama Chilena de la AAP el Prof. Raúl Gantes ${ }^{6}$, quién en ese momento era también el Secretario de Redacción de la Revista Chilena de Pediatría (RCHP). Ese año aparece nuestro logo por primera vez en la RCHP, precediendo el índice general del volumen XXVII (figura 3). Destacable es que gran parte de las presentaciones internacionales que se efectuaban por parte de los profesores pediatras nacionales en diversos países, llevaban incorporados el logo o alguna de sus variantes. En 1999, con el traslado de la sede institucional a su ubicación actual, se establece el fondo tricolor definitivo para el logo, como una forma de darle un carácter de orientación más nacional, diseñándose la insignia que ornamenta actualmente el frontis de la sede. El año 2003 se unifica el diseño, muy variado hasta entonces (figura 4), con el año de la fundación en la base y la frase "sociedad chilena de pediatría” sobre el arco superior (Actas de Reuniones de Directorio).

Detrás de un logo, existen organizaciones; tras las organizaciones existen propósitos e intenciones; tras éstas existen personas, y tras las personas una historia. Este artículo pretende aclarar la historia tras un logo, para intentar entender el porqué de su elección por parte de aquellos 29 pediatras visionarios que, reunidos

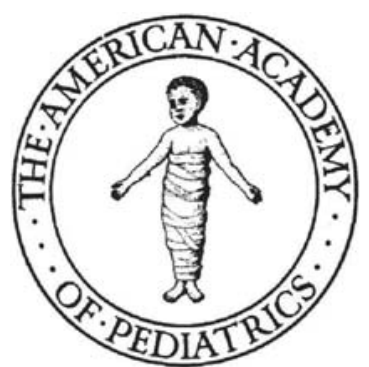

AAP, 1930

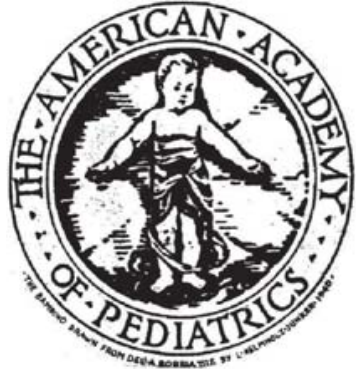

AAP, 1955

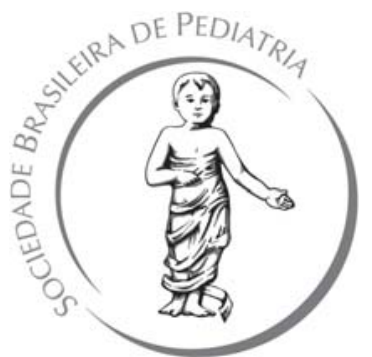

Brasil, 1936
Figura 2. Primer logotipo de la AAP en 1930 y el definitivo de 1955, y el logo de la Sociedad Brasileña de Pediatría. 

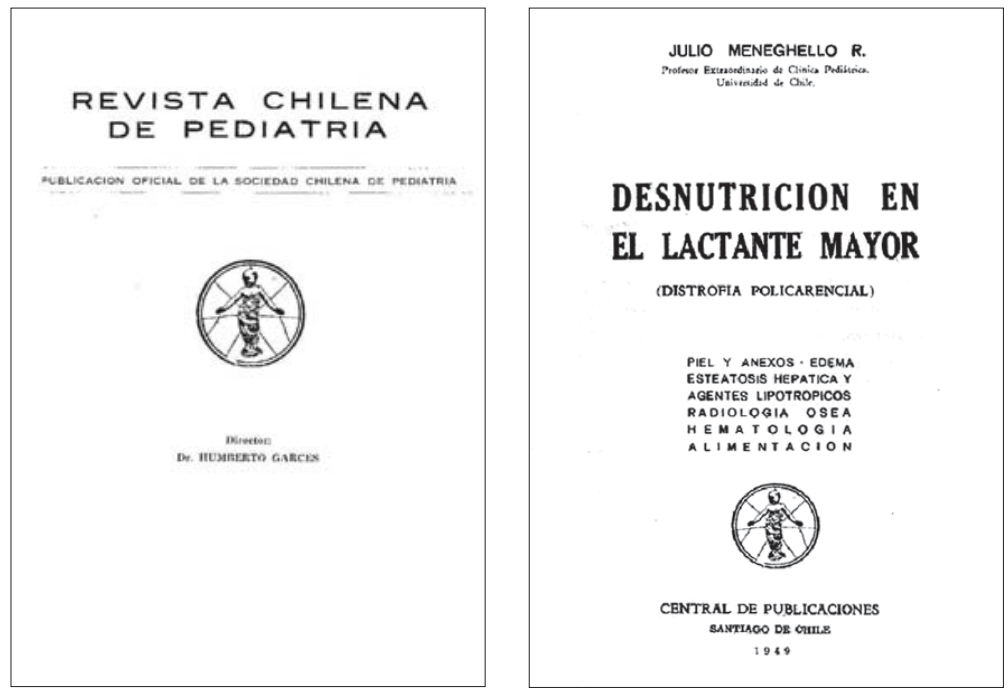

Figura 3. Primera aparición del logo en la Revista Chilena Pediatría y variantes del logo en publicaciones internacionales, como las del Prof. Dr. Meneghello en 1949 y en Lima 1957 (V Congreso Panamericano de Pediatría y de Sociedades Panamericanas de Pediatría).

por Calvo Mackenna, unieron sus propósitos e intenciones en pro de la salud de niños y adolescentes, fundando las bases de nuestra Sociedad Chilena de Pediatría 6 .

\section{El hospital de los Inocentes}

(Lo Spedale o L'Ospedale di Santa María degli Innocenti)

Los hitos que marcan el inicio del Renacimiento europeo son la caída del Imperio bizantino, la toma de Constantinopla por los turcos (1453) y el surgimiento de las primeras nacionalidades; entre las nuevas condiciones tecnológicas, la invención de la imprenta (1474) que derriba la barrera a la difusión de la cultura, la brújula (1302) que vence la barrera que impone la geografía, y la pólvora (1324) que vence la barrera que imponen los feudos. Sin embargo, como fenómeno social, es la visión antropocéntrica de la vida la que destaca este período, ya que el ser humano es considerado el centro del quehacer divino y no un instrumento de paso como era la visión feudalista medioeval, que restaba importancia al hombre, en especial al niño, cuya muerte o sufrimiento hasta entonces, era considerada una situación natural ${ }^{8,9}$.

El Hospital de los Inocentes (figura 5) es una de las construcciones más famosas y visita obligada en Florencia, Italia. Nace como una organización dedicada a acoger a niños abandonados, cuyo nombre evoca a "los Inocentes", los niños de Belén menores de dos años, mandados a asesinar por Herodes cuando se entera que ha nacido el futuro rey de Israel (Mateo 2: 16-18). El origen del apellido Innocenti y sus variantes deriva de instituciones como la del Ospedale, que a partir de éste, se extenderían por toda Europa.

El 19 de Marzo de 1294, el Consejo General de la entonces República Florentina nomina al Patronato de los Mercaderes de la Seda (L'Arte della Seta), como protectores y educadores de los niños abandonados de la ciudad ${ }^{8}$. Siguiendo este mandato, el 8 de Abril de 1419 el Patronato da inicio a la construcción de este Ospedale, con un presupuesto inicial de 1000 florines donados por el comerciante y filántropo Francesco di Marco Datini ${ }^{9,10}$. Llama la atención que se eligiera deliberadamente a una institución laica en vez de una organización religiosa o a la Iglesia, probablemente debido a la orientación de compromiso social, humanitario, que se le da a la protección de la infancia en ese momento, más allá de una obra de caridad o propio de una institución ${ }^{10}$. Ubicado junto a y formando parte de la plaza de la Santísima Annunziata (1252), se establece su dedicación exclusiva para al cuidado de estos niños 


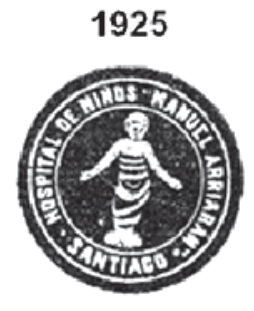

2000

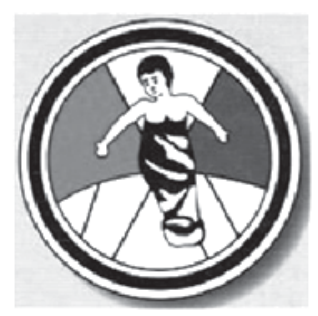

1955

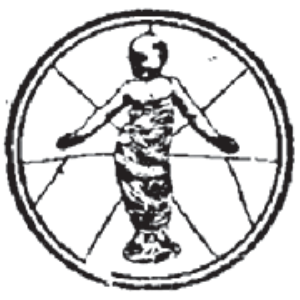

2002

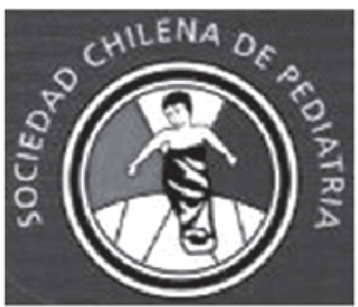

2003

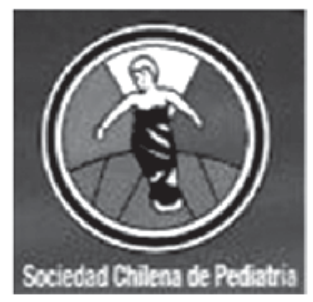

1957

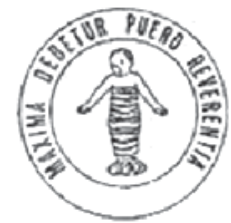

2004 adelante

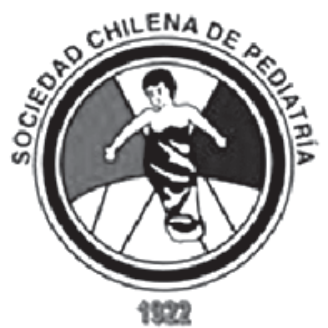

Figura 4. Variantes del logo y logo actual.

"...quienes son comúnmente llamados expósitos... cuyos padres y madres, contra la ley y la naturaleza humana, los han abandona$d o^{\prime 10}$. Filippo di Ser Brunelleschi Lippi, conocido como Brunelesco, (1377 - 15 de Abril 1446) miembro de este patronato desde 1404, hizo el diseño y dirigió personalmente la obra como "capomaestro" hasta 1427, abandonando el esquema del antiguo hospital medioeval dando origen a un nuevo modelo, el que se transformó en el ejemplo de la construcción renacentista. Posterior a 1427, Francesco della Luna se hace cargo de las obras, a quien se ha atribuido el

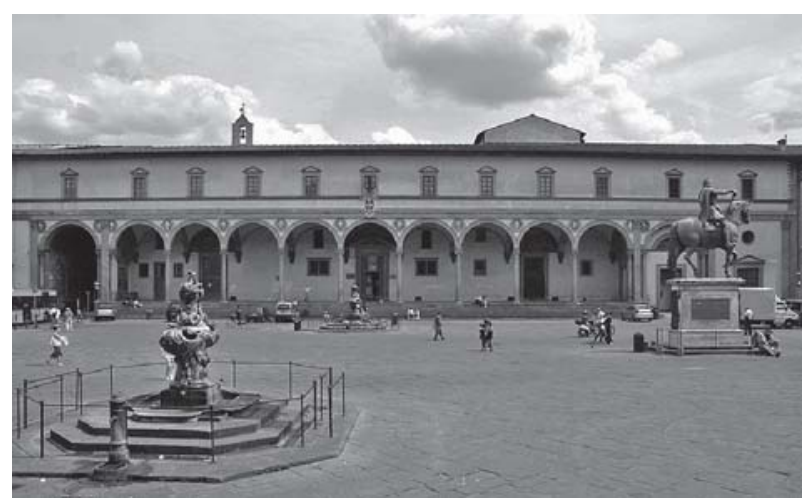

Figura 5. Hospital de los Inocentes. segundo piso de la fachada, finalizando Jacopo Rosselli ${ }^{7}$. Su construcción se termina e 24 de Enero de 1445, año en que inicia sus funciones con la acogida de los primeros 62 niños $^{8}$, la primera de las cuales ingresa el 5 de Febrero de 1445, para Santa Ágata, por lo que fue bautizada como Ágata Smeralda. En los años siguientes, el número de niños que recibió fue significativo: en 1454 se contaba con 260 niños, en 1511 con 1200 y para 1660 se refiere entre 3000 y 4000 incluyendo a quienes les cuidaban, educaban y protegían ${ }^{10,11}$.

En el pórtico, a base de arcos, se refleja el sistema de proporciones en que se basa la arquitectura brunellesquiana, pues el ancho del vano y del pórtico es igual al alto de las columnas, con lo cual se forma un cubo que se repite nueve veces $^{9}$, ofreciendo una amplia comunicación al exterior y la plaza. Esta disposición lo diferencia de los “ospedali”, la visión medioeval del hospital, más cercano a los monasterios, cerrados, con escasa circulación de gente, en contraposición con esta característica de apertura más cercana a la concepción actual del hospital, con una amplia comunicación al exterior a través de los arcos del pórtico o “loggia”, 
que permite el libre desplazamiento de familiares, visitantes y trabajadores dedicados al cuidado de los niños en forma tal que el pórtico se comunica e integra en un todo con la plaza en la que se ubica; a su vez, permite que el niño tenga amplios espacios de esparcimiento y contacto con el exterior, desarrollándose el concepto de "ospedale", o su diminutivo "spedale"11,12. Este cambio es concordante con las corrientes de pensamiento de la época que ya empezaban a distinguir la condición de ser independiente, con derechos propios, a los niños y adolescentes $^{8-10}$.

Las columnas son de tipo corintias y adornadas, en cada espacio que dejan los arcos, con medallones en terracota policroma ejecutados por Andrea Della Robbia entre 1463 y 1466, que representan a bebés-querubines en actitud de súplica, que visten simbólicamente la ropa elaborada por quienes eran los encargados de velar por los niños abandonados en ese entonces, los mercaderes de seda de Florencia, a la usanza de la época, siendo éstos la principal obra del artista ${ }^{11,12}$. Probablemente sólo los 10 medallones centrales son su obra, fueron montados recién en 1487, cuarenta años después de la muerte de Brunelleschi. Los restantes 4, dos pares en los extremos de la loggia, fueron instalados en 1845, durante ampliaciones posteriores ${ }^{13,14}$. En el extremo derecho del pórtico se ubicaba una especie de cuenca próxima a una ventana amplia que comunicaba a una sala, donde una dama hacía turno esperando la llegada de algún niño abandonado, cuyo primer llanto avisaba su presencia; este sistema duró hasta 1660, año en que se construye la famosa "rueda" o "torno" en la que eran depositados los niños, ubicada ahora en el extremo izquierdo del pórtico, sistema que dura hasta 1875 . La inscripción, en lo alto de este pórtico, corresponde al Salmo 26 de la Biblia en latín y dice "Pater et mater reliquerunt nos, Dominus autem assumpsit", o sea "nuestros padres y madres nos han abandonado, el Señor al contrario nos ha acogido"10,11. Al girar esta rueda, el niño ingresaba a un mesón en el que la imagen de José y María dejaban el espacio exacto en que encajaba este mecanismo haciendo que, al menos por unos instantes, el menor abandonado ocupara el lugar de Jesús-niño ${ }^{8,11,12}$.
Muchos artistas, entonces famosos, contribuyeron a su construcción y ornamentación, como Luca y Andrea della Robbia, il Rossellino (1427-1478/1481), Filippo Lippi (1406-1469), Giuliano da Sangallo (1445-1516), Piero di Cosimo (1462-1521) y Domenico Ghirlandaio (1449-1494), quien expone su obra maestra, la Adoración de los Magos (1488), como homenaje de la ciudad completa hacia los niños ${ }^{13}$.

Finalizado el Patronato en 1775, fue administrado hasta 1814, por una Comisión especial para el Hospital, creada por el gobierno francés. Desde 1862 su administración se separa del gobierno imperante quedando en manos de un Concilio Administrativo operativo hasta 1888, año en el cual de procede a la elección de un Secretario General. Desde 1890 adquiere la figura de Instituto de Asistencia Pública y Beneficiencia, administrado por un Concilio en que concurren representantes de la región Toscana, de la Provincia y de la comuna de Florencia, dirigida por un Presidente ${ }^{4,11}$. Posterior a la inundación de 1967, fue restaurado completamente gracias a aportes de múltiples instituciones, devolviéndole muchas de sus características originales. Desde 1988 alberga al Centro de Investigación Innocenti, principal entidad investigativa del UNICEF (creada en 1947), fundado para mejorar los conocimientos de los derechos de la infancia a nivel internacional y promover la aplicación de la Convención sobre los Derechos del Niño (1959) en los países industrializados y en desarrollo ${ }^{14}$. Desde el 2002 una reforma de los estatutos ha permitido crear la figura de un Director General. En la actualidad funciona también un Museo histórico sobre éste tópico y de arte relacionado a la niñez.

Con el nombre de la primera niña recibida en L'Ospedale, Ágata Smeralda, se creó en 1991, en Brasil, tras la visita del Papa Juan Pablo II a ese país, una ONG de proyección internacional, cuya sede reside actualmente en Florencia desde 1992, encargada de velar por los niños abandonados y víctimas de la violencia; desde Brasil a Costa de Marfil, India, Congo, Israel, Jordania, Nigeria, Sri Lanka, Albania. Especialmente en Brasil, donde se encarga de la protección de niños con discapacidad (no videntes, parálisis cerebral), hijos de detenidos y madres presidiarias, niños víctimas de abuso 
o violencia, abandonados y en riesgo biosocial, a través de acciones directas, apoyando diversas instituciones y facilitando su reinserción familiar, social o adopción (www.agatasme ralda.org).

Así, el Hospital de los Inocentes constituye todo un hito desde varios aspectos. Primero, es la más antigua construcción conocida dedicada específicamente y en forma continua al cuidado, educación y formación de niños. En segundo lugar, los historiadores de la arquitectura lo identifican como la primera estructura verdaderamente renacentista, siendo además la primera comisión de Brunelesco, el extraordinario arquitecto, matemático e ingeniero del naciente Renacimiento italiano. Adicionalmente, la construcción en si misma y el como se gestó su inicio, representan un hito social y cultural fundamental del Humanismo durante el "quattrocento" que hace concreto el pensamiento renacentista. Además, queda su proyección en organizaciones que promueven, defienden y velan por los derechos de los niños, como el Centro de Investigación Innocenti (UNICEF) y la Fundación Ágata Smeralda, entre muchos otros.

\section{El "Bambino" o el "Innocenti"}

Andrea Di Marco Di Simone Della Robbia (20 ó 24 de Octubre 1435, Florencia - 4 de Agosto de 1525, Florencia), es adoptado por su tío Luca Della Robbia (1399-1482), famoso escultor florentino, junto a sus cinco hermanos cuando, en 1448, su padre, Marco, fallece prematuramente. Andrea es el principal seguidor de la actividad de Luca que le enseña el arte de la escultura y todos los secretos de la tierra cocida, hoy conocida como terracota vidriada, debido a un proceso de cocido y esmaltado inventado por Luca. Mientras Andrea trabaja en el taller familiar, recibe un sinfín de encargos que le convierten en el verdadero protagonista del mismo; ese protagonismo provoca los celos de su tío que lo deshereda en su testamento (19 de febrero de 1471) porque considera que el joven ya posee bastante riqueza pues ha aprendido "un arte muy remunerativo, fruto de su ingenio y de sus enseñanzas, que le aporta fáciles honores y notables ganancias ". Sin embargo, a la muerte de su tío en 1482, se hace cargo de la familia y el taller. Su principal trabajo (1463 - 1466), fueron los tondos o rodelas de terracota que adornan el Hospital de los Inocentes (figura 6) y que se han convertido en el símbolo del humanismo renacentista y de la percepción a los niños como sujetos de derecho ${ }^{15,16}$.

Andrea tuvo varios hijos, de quienes Giovanni (1469-1529/1530), Girolamo (1488-1566), Luca el Joven (1475-1550), Marco (1458-1534) y Pierfrancesco (1477-1528) son los más conocidos. Giovanni ejecutó las relevaciones famosas para el Spendale del Ceppo, Pistóia y quién mantuvo en funciones el taller posterior a la migración de sus hermanos a Francia ${ }^{16}$.

Las teorías sobre el diseño mismo que Andrea realizó para la confección de los "bambini”, sus motivaciones y la simbología que proyecta son parte de la historia desconocida de esa época; sin embargo, se puede destacar el aspecto angelical de las formas, el aparente buen estado nutricional que destacan y el gesto amable en la mirada junto al de solicitud expresada en sus brazos. Se puede especular que representan a Jesús-niño, expresando gestos de confianza en quienes lo están acogiendo y protegiendo, pero a la vez de solicitud de apoyo ante el abandono. Las libertades creativas de Della Robbia se observan también en el vestuario; los ropajes que cubren al niño son el reflejo de la moda de la época que, con distintos argumentos, se usaba envolver a los menores
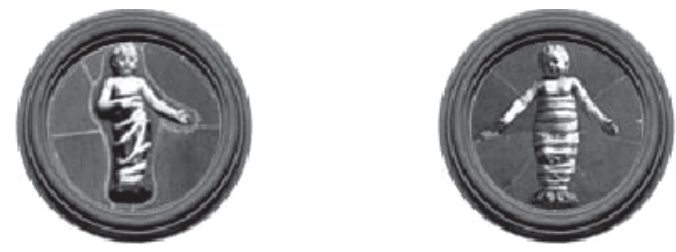

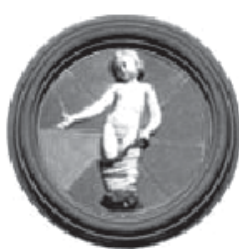

Figura 6. Tondos o Rodelas de terracota representando al Bambino expósito. 
de tres meses por completo, dejando sólo la cabeza al descubierto ${ }^{10,17}$; era raro que los niños mayores siguieran fajados. Desde los tiempos bíblicos (Lucas 2:7) que se destaca la usanza de envolver a los niños para "protegerlos de las inclemencias del tiempo, de los desechos fisiológicos y favorecer su crecimiento"8,9. Sin embargo, el bambino aparenta más de tres meses, pareciera de alrededor de 12 a 18 meses y la expresividad y gestualidad también representan a un niño mayor. Como sea, Andrea Della Robbia consigue el propósito de obtener la simpatía, encantamiento, por parte del observador y reflejar la importancia que adquiere el niño en el naciente Humanismo renacentista, destacándole características que le confieren atributos de "persona": expresividad, sentimientos y necesidades $^{17,18}$.

\section{Conclusiones}

Puede cerrarse esta historia con la frase de Décimo Junio Juvenal (55-138 DC), célebre moralista y escritor latino, la que en algún momento formó parte de nuestra insignia: "Maxima debetur puero reverentia"," todo niño merece el máximo respeto”, (Sátiras, XVI, 47), frase en la que se inspiraron los pensadores del Renacimiento, que retoman el conocimiento greco-romano en su visión del hombre. Historia que trata del rescate del concepto de protección a la infancia, que lentamente se extiende por Occidente a partir de $L^{\prime}$ Ospedale $e^{19,20,21}$.

Esta es la historia detrás de un logotipo, el que tiene el propósito de representar aquellos valores que nuestros predecesores, fundadores de la Sociedad Chilena de Pediatría reunidos por el Prof. Luis Calvo Mackenna hace 86 años, tuvieron como ideario, visión y misión: proteger a la infancia. Las formas de lograr este propósito, característica de la pediatría, pueden ser muchas. Sin embargo, si no se piensa en los niños y adolescentes como sujetos de derecho, como continuadores de un proyecto histórico humano, independiente, aunque complementario con puntos de vista espirituales, toda acción en su favor puede verse como una simple obligatoriedad contractual o acciones paliativas. Se requiere de una acabada forma- ción humanista e integral para que se nos permita comprender todo el panorama de la niñez y su evolución para enlazarlo con un destino y una meta históricos ${ }^{22,23}$. Ese es el mérito de L'Arte della Seta, del pensamiento humanista característico del Renacimiento, que se traduce en políticas y acciones concretas decididas a proteger a los niños y que Della Robbia representa magníficamente en sus "tondos".

Así, a toda la capacidad organizativa y los méritos académicos que demostraron aquellos 29 ilustres pediatras Fundadores, debe sumarse, en justicia, el gran mérito de haber elegido una insignia con tan importante simbolismo, haciendo de la historia del logo la historia del desarrollo de una capacidad humana fundamental: el amor por los niños y nuestros semejantes.

\section{Agradecimientos}

Mis agradecimientos al Dr. John Zwicky, Director del Pediatric History Center de la American Academy of Pediatrics por facilitar el acceso a sus archivos históricos.

\section{Referencias}

1.- http://www.neatorama.com/2008/02/07/the-evolutionof-tech-companies-logos/ (visitada el 26 de Febrero 2008).

2.- http://www.logoorange.com/logodesign-A.php (visitada el 26 de Febrero 2008).

3.- Vargas $N$ : Historia de la Pediatría en Chile: crónica de una alegría. Ed. Universitaria, primera edición, Santiago de Chile, 2002.

4.- Kahn L: The "Ospedale degli Innocenti" and the "Bambino" of the American Academy of Pediatrics. Pediatrics 2002; 110: 175-80.

5.- Beaven P. The origin and significance of the Academy’s della Robbia insignia. Pediatrics 1956; 17: 765-9.

6.- Aristía A: La sección latinoamericana de la AAP: El Distrito IX. Rev Chil Ped 1957; 28: 468-9.

7.- Barrera F, Moraga F, Escobar S, et al: Participación de la madre y la familia en la atención del niño hospitalizado: análisis histórico y visión de futuro. Rev Chil Ped 2007; 78: 85-94.

8.- Gavitt P: Charity and children in Renaissance Florence. Ann Arbor, MI: The University of Michigan Press, 1990 
9.- Hornik H: Protecting de Innocents. Hospitality 2007; 64-66. (www.baylor.edu/content/services/document. php/53386.pdf visitada el 26 de Febrero 2008)

10.- Viazzo P, Bortolotto $M$, Zanotto A: Abandoned Children. En: Panter-Brick C, Smith M, eds. Five Centuries of foundling history in Florence: Changing patterns of abandonment, care and mortality. Cambridge, UK: Cambridge University Press; 2000.

11.- http://www.unicef-irc.org/aboutirc/spedaledeglinno centi.html (visitada el 26 de Febrero 2008).

12.- Argan G: The architecture of Brunelleschi and the origins of perspective theory in the Fifteenth Century, J. Warburg and Courtauld Institutes 1946; 9: 96-121.

13.- Baron J: Art in hospitals. J R Coll Phys 1995; 29: 131-44.

14.- http://www.unicef.org/spanish/research/4276.html (visitada el 26 de febrero de 2008).

15.- http://www.ebrisa.com/portalc/articulo-B/411934, Familia della Robbia, Enciclopedia Británica-Salvat on line: (visitada el 26 de Febrero de 2008).

16.- http://mx.encarta.msn.com/encyclopedia_761565576/ Robbia_(familia).html, Familia Robbia, Enciclopedia
Encarta on line. (visitada 26 de Febrero 2008).

17.- http://www.histgueb.net/expositos/registros.htm (visitada 26 de Febrero 2008).

18.- Pita A: Historia de la Verdad en Medicina. Talleres Gráficos Argentinos, Bs As, 1947.

19.- Lamas T: Historia de la Medicina: hechos y personajes. Edit. Mediterráneo, primera edición, Santiago de Chile, 2004.

20.- De Tezanos Pinto S: Breve historia de la Medicina Universal y notas sobre historia de la Medicina chilena. Edit. Universitaria, segunda edición, Santiago de Chile, 1987.

21.- Buford N, Ballabriga A, Kretchmer N: editores. History of Pediatrics 1850-1950. Nestlé Nutrition Workshop Series 22. Raven Press, New York, 1991.

22.- Schonhaut L: El rol de la Pediatría del Desarrollo en el control de salud integral: hacia la Pediatría del siglo XXI. Rev Chil Pediatr 2004; 75: 9-12.

23.- Moraga F, Saieh C, Quezada A: Respuestas a la audiencia con el Comité Asesor Presidencial de Políticas para la Infancia. Rev Chil Pediatr 2007; 78 supl 1: 51-6. 